\title{
Surgical Treatment of Spinal Cord Compression Caused by Metastatic Small Cell Lung Cancer: Ten Years of Experience in a Single Center
}

This article was published in the following Dove Press journal: Cancer Management and Research

\author{
Xin Gao ${ }^{1, *}$ \\ Kun Zhang ${ }^{1, *}$ \\ Shuang $\mathrm{Cao}^{1,2, *}$ \\ Shuming Hou',* \\ Tao Wang' \\ Wen Guo (iD) ${ }^{1,3}$ \\ Zheyu Wu ${ }^{1,4}$ \\ Qi Jia' \\ Tielong Liu' \\ Jianru Xiao' \\ 'Orthopaedic Oncology Center, \\ Department of Orthopedics, Changzheng \\ Hospital, Second Military Medical \\ University, Shanghai, People's Republic of \\ China; ${ }^{2}$ Department of Orthopedics, \\ Third Affiliated Hospital of Anhui Medical \\ University, Hefei, Anhui Province, \\ People's Republic of China; ${ }^{3}$ Department \\ of Orthopedics, Taizhou People's \\ Hospital, Taizhou, Jiangsu Province, \\ People's Republic of China; ${ }^{4}$ Department \\ of Orthopedics, Zhongnan Hospital of \\ Wuhan University, Wuhan, Hubei \\ Province, People's Republic of China \\ *These authors contributed equally to \\ this work
}

Purpose: Metastatic spinal cord compression (SCC) secondary to small cell lung cancer (SCLC) is a disastrous oncological emergency, but it is poorly understood due to the small numbers of patients and their short survival times. Whether patients suffered from SCC caused by metastatic SCLC benefit from spinal surgery remains unknown. The aim of this study was to evaluate the role of surgical treatment and prognostic factors in patients with SCC caused by metastatic SCLC.

Methods: From 2009 to 2019, 30 consecutive patients surgically treated for metastatic SCC from SCLC were enrolled in this retrospective analysis. Kaplan-Meier method and Cox regression analysis were used to estimate overall survival (OS) and identify prognostic factors. Quality of life (QoL) was assessed by the three-level EuroQol-five-Dimensions (EQ-5D-3L) instrument and compared using Student's $t$ test.

Results: The median OS time was 9 months in our series. Relief of pain, preservation of neurological function, and improvement of performance status were achieved after surgical intervention. The mean EQ-5D-3L utility score showed a significant improvement after surgery ( 0.3394 preoperatively vs 0.5884 postoperatively). According to Cox regression analysis, postoperative ECOG-PS and immunotherapy were identified to be independent prognostic factors for patients with SCC caused by metastatic SCLC.

Conclusion: Despite the short life expectancy, prompt surgical decompression is extremely necessary for patients with SCC caused by SCLC, for surgery played a critical role in improving patients' QoL. Better performance status after surgery and receiving immunotherapy were associated with a longer OS.

Keywords: small cell lung cancer, spinal cord compression, surgery, quality of life, prognostic factor, immunotherapy

\section{Introduction}

Small cell lung cancer (SCLC) is a lethal tumor accounting for approximately 15\% of all lung cancers. ${ }^{1}$ Comparing with other types of lung cancer, SCLC is characterized by a more rapid doubling time, a higher growth fraction, and earlier development of widespread metastases, ${ }^{2}$ with around two-thirds of SCLC patients presenting with metastatic disease at diagnosis. ${ }^{3}$ Despite the chemosensitivity of SCLC, most patients relapse within a year after the initial treatment. ${ }^{4}$ Prognosis in SCLC is poor. Median survival time has been reported as 23 months for patients with limited-stage disease, 7-12 months for patients with extensive-stage disease, and only 2-4 months for patients without treatment. ${ }^{3,4}$ It is estimated that there
Correspondence: Tielong Liu; Jianru Xiao Orthopaedic Oncology Center, Department of Orthopaedics, Changzheng Hospital, No. 415 Fengyang Road, Huangpu District, Shanghai,

People's Republic of China Tel +0086-02I-8I886843

Fax +0086-021-63520020

Email liutielongvip@I63.com; xiaojianruvip@I63.com 
were 250,000 cancer deaths caused by SCLC worldwide yearly, leading SCLC to be a worldwide serious public health problem. ${ }^{1}$

Bone metastasis was found in $27-41 \%$ of SCLC patients at initial presentation. ${ }^{5}$ Among patients with bone metastases of SCLC, $70.5 \%$ had spinal metastases. ${ }^{6}$ Spinal cord compression (SCC) is a disastrous complication of spinal metastatic tumor, leading to a substantial and striking change in quality of life (QoL) for patients. ${ }^{7,8}$ The role for surgical intervention for metastatic SCC is well established with the goal of pain relief, preservation of neurologic function, maintenance of spinal stability, and improving QoL. ${ }^{9,10}$ Our experience in surgical management of non-small cell lung cancer (NSCLC) spinal metastasis showed that complete disappearance of deficits in spinal cord function after surgery was the most robust favorable prognostic factor of survival, ${ }^{11}$ and surgical treatment significantly improved the QoL over the nine-month assessment period. ${ }^{12}$ However, little is known about spinal metastasis of SCLC, because there is a lack of studies due to small numbers of patients and their short survival times. The largest isolated cohort of this complicated disease was reported by Goldman et al in $1989 .{ }^{13}$ In their study, 24 cases of SCC caused by SCLC were identified from 610 cases of SCLC, and most of them were treated by radiotherapy. The authors emphasized the poor prognosis of this condition, and early prophylactic radiotherapy was also encouraged. Nevertheless, whether patients suffered from SCC caused by metastatic SCLC, such a highly malignant tumor with short life expectancy, benefit from spinal surgery remains unknown.

In this study, we retrospectively reviewed 30 consecutive patients with SCC from metastatic SCLC treated with urgent surgery over a period of 10 years, to provide some useful insight into clinical characteristics, surgical treatment, outcomes, and prognostic factors on this kind of challenging disease.

\section{Methods}

A retrospective review was performed of 30 consecutive patients surgically treated for metastatic SCC secondary to SCLC in our spine tumor center between January 2009 and January 2019. This study was approved by the ethics committee of Changzheng Hospital, confirmation of patient written informed consent was obtained from all patients. All procedures performed in studies involving human participants were in accordance with the Declaration of Helsinki.

The clinical and operative records, radiographic images, and pathological reports of all 30 patients were reviewed by two individual researchers. Visual Analogue Scale (VAS), Frankel Grade, and Eastern Cooperative Oncology Group performance score (ECOG-PS) were used to evaluate the degree of pain, neurological status, and performance status, respectively. Positron emission tomography-computed tomography (PET-CT) was done to find possible metastatic sites.

The surgical decision-making was made on the guidance of NOMS framework, ${ }^{14,15}$ SINS classification system, ${ }^{16}$ and Bilsky epidural SCC scale. ${ }^{17}$ Generally, indications for surgery were neurologic deficits caused by SCC, spinal instability or a combination of these, and all operative patients were considered to have the ability to tolerate the proposed intervention based on the extent of systemic comorbidities and tumor burden, and a life expectancy of more than 3 months. The surgical protocol was circumferential decompression of the spinal cord, tumor excision, reconstruction and stabilization of the spine. Cisplatin dissolved in distilled water was applied to soak the surgery field for intraoperative chemotherapy except when the dura was broken.

Clinical management of metastatic SCC caused by SCLC requires a multidisciplinary approach which integrates surgeons, oncologists, radiotherapists and histologists, etc. The combination of cisplatin and etoposide was used as first-line chemotherapy. Postoperative radiotherapy was performed for local control of the residual tumor. Bisphosphonate (zoledronic acid) was administered to help prevent skeletal-related events. Immunotherapy (PD1 immune checkpoint inhibitor, pembrolizumab, nivolumab, and toripalimab) was also selected on the basis of personalized evaluation. Chemotherapy, radiotherapy, bisphosphonate therapy, and immunotherapy were performed by our multidisciplinary team.

Patients were followed up at 2, 6, and 12 months after surgery, every 6 months for the next 2 years, and once a year thereafter. Overall survival (OS) was defined as the interval between the date of the spinal surgery for metastatic SCLC in our center and the date of death or until June 2019 for alive patients. The last status of patients was obtained from office visit or telephone interview.

Patient-reported QoL was evaluated by the three-level EuroQol-five-Dimensions (EQ-5D-3L) instrument, which is one of the most frequently applied QoL measurements with five dimensions (mobility, self-care, usual activities, pain/discomfort, and anxiety/depression). The Chineselanguage version of the EQ-5D-3L questionnaire was administered before surgery and at 2-months follow-up for our patients. All EQ-5D-3L questionnaire data were 
collected and checked by two individual researchers, and missing data were minimized through telephone calls. The overall EQ-5D-3L utility scores (range -1 to 1 ) were calculated based on the Chinese utility values, ${ }^{18}$ in which an overall utility score of zero represents death, 1 represents full health, and a negative value indicates that the health state may be even worse than death.

All statistical calculations were performed by SPSS Statistics, version 22.0 (IBM corp., New York, USA). The Kaplan-Meier method was adopted to estimate the OS time, with Log-rank test to identify the difference. Factors with $\mathrm{P}$ values less than 0.05 were considered statistically significant and subjected to multivariate analysis using the Cox proportional hazards model to further identify factors that independently predicted survival. Comparison between preoperative EQ-5D-3L utility scores with utility scores at 2-months follow-up was conducted by Student's $t$ test. All tests of significance were two-sided, and $\mathrm{P}<0.05$ was considered statistically significant.

\section{Results}

\section{Patient Descriptions}

The characteristics of 30 patients are shown in Table 1 . The series was comprised of 26 men and 4 women, with a mean age of 60.8 years (median 61.5, range 30 to 80 ). Before finding their primary foci in lung, $24(80 \%)$ patients identified metastatic disease in the spine initially with the common symptom of persistent back or radiative pain. Notably, 13 (43\%) patients presented with incomplete paralysis before surgery. SCC of 4 patients located in the cervical spine, 15 patients in the thoracic spine, 11 patients in the lumbar spine. Vertebral tumors were identified in 21 patients, and intraspinal tumors were observed in 9 patients (5 extramedullary-intradural and 4 intramedullary). All patients with vertebral tumor scored higher than 7 in SINS system (range 8 to 17), and the scores of 8-12 and 13-17 were documented in 9 and 12 patients, respectively. According to Bilsky epidural SCC score, all patients with vertebral tumor were classified as Grade 2.

\section{Treatment}

All patients in our series received urgent surgical treatment within 72 hours after diagnosis of SCC. Total resection of spinal tumor was performed in 22 patients, while 8 patients underwent subtotal resection. Postoperative radiotherapy was further performed in 12 patients. For the primary lung tumor, surgery and radiotherapy were performed in 4 and 3 patients, respectively. Systematic chemotherapy was prescribed to all patients, but 2 of them failed to receive chemotherapy due to the poor general condition. 20 patients were treated with bisphosphonate to inhibit osteolysis. After assessment by oncologists and pulmonary physicians, 11 patients further received immunotherapy of PD-1 immune checkpoint inhibitor.

\section{Follow-up and Outcomes}

Postoperative complication was observed in one patient who had surgical site infection and recovered after debridement and antibiotic therapy. No surgical-related perioperative death occurred in this series, but one patient died 1 month after surgery due to rapid progression of the primary lung cancer. For other 29 patients, all had substantial pain relief after spinal surgery. The mean VAS score dropped from 7.2 (range 4 to 10) preoperatively to 2.9 (range 1 to 5) postoperatively. 10 (34\%) patients had an improvement of neurological function in their 2-month follow-up. Frankel Grade showed 1-grade improvement in $9(31 \%)$ cases and 2-grade improvement in $1(3 \%)$ case. The general performance status of patients was also improved at least 1-grade of ECOG-PS in 9 (31\%) patients, and the rate of patients with ECOG-PS of 0-2 raised from $52 \%$ ( 15 cases) to $69 \%$ (20 cases).

The mean follow-up duration was 11.8 (range 1 to 68 ) months for all patients. $25(83 \%)$ patients died with a mean period of 10.5 months (range 1 to 68 ) between observing spinal metastasis and death, while $5(17 \%)$ patients are still alive with a mean survival time of 18 months (range 6 to 46). According to Kaplan-Meier curve, the 1-year survival rate in all patients was $29.1 \%$, with the median OS time of 9 (95\% CI 4.2-13.8) months. Kaplan-Meier curve of OS for all 30 patients is shown in Figure 1A.

\section{QoL Assessment}

Among the five dimensions of the EQ-5D-3L, health problems in pain/discomfort improved most frequently, followed by anxiety/depression, mobility, self-care, and usual activities. The mean EQ-5D-3L utility score was 0.3394 (median, 0.339 ; range -0.149 to 0.795 ) before surgery, while the mean utility score at 2-month follow-up rose up to 0.5884 (median, 0.604; range 0.22 to 0.887 ), and the difference was statistically significant $(\mathrm{P}<0.001)$. Notably, the preoperative EQ-5D-3L utility score was less than zero in 6 patients $(20 \%)$, emphasizing the terrible condition for patients with SCC caused by SCLC. 
Table I Patient Characteristics and Univariate Analysis of the Prognostic Factors Affecting OS

\begin{tabular}{|c|c|c|c|}
\hline \multirow[t]{2}{*}{ Factors } & \multirow[t]{2}{*}{$\mathbf{N}$} & \multicolumn{2}{|l|}{ OS } \\
\hline & & Median (m) & $\mathbf{P}$ \\
\hline Patient-related factors & & & \\
\hline Sex, M/F & $26 / 4$ & $9 / 6$ & 0.243 \\
\hline Age, $\leq 60$ y/>60 y & $12 / 18$ & $12 / 5$ & 0.339 \\
\hline Smoker, no/yes & $9 / 21$ & $6 / 10$ & 0.310 \\
\hline Onset symptom, pain/other & $23 / 7$ & $9 / 9$ & 0.324 \\
\hline $\begin{array}{l}\text { Duration of symptoms, } \leq 2 \mathrm{~m} / \\
>2 \mathrm{~m}\end{array}$ & $15 / 15$ & $9 / 9$ & 0.611 \\
\hline $\begin{array}{l}\text { Preoperative Frankel Grade, A-C/ } \\
\text { D-E }\end{array}$ & $13 / 17$ & $4 / 9$ & 0.655 \\
\hline Preoperative ECOG-PS, 0-2/3-5 & $16 / 14$ & $10 / 4$ & 0.285 \\
\hline Comorbidity, no/yes & $18 / 12$ & $8 / 10$ & 0.821 \\
\hline $\begin{array}{l}\text { Postoperative Frankel Grade, A-C/ } \\
\text { D-E* }\end{array}$ & $8 / 21$ & $4 / 10$ & 0.161 \\
\hline Postoperative ECOG-PS, 0-2/3-5* & $20 / 9$ & $12 / 3$ & 0.022 \\
\hline Tumor-related factors & & & \\
\hline Tumor size, $<3 \mathrm{~cm} / \geq 3 \mathrm{~cm}$ & $11 / 19$ & $9 / 10$ & 0.596 \\
\hline Tumor site, vertebral/intraspinal & $21 / 9$ & $10 / 8$ & 0.631 \\
\hline $\begin{array}{l}\text { Number of spinal lesions, single/ } \\
\text { multiple }\end{array}$ & $24 / 6$ & $9 / 2$ & 0.868 \\
\hline $\begin{array}{l}\text { Extraspinal bone metastasis, no/ } \\
\text { yes }\end{array}$ & $25 / 5$ & $9 / 5$ & 0.117 \\
\hline $\begin{array}{l}\text { Extrapulmonary visceral } \\
\text { metastasis, no/yes }\end{array}$ & $23 / 7$ & $9 / 5$ & 0.262 \\
\hline Treatment-related factors & & & \\
\hline Surgery for lung cancer, no/yes & $26 / 4$ & $8 / 12$ & 0.429 \\
\hline $\begin{array}{l}\text { Radiotherapy for lung cancer, no/ } \\
\text { yes }\end{array}$ & $27 / 3$ & $9 / 5$ & 0.500 \\
\hline Preoperative embolization, no/yes & $22 / 8$ & $8 / 9$ & 0.513 \\
\hline Resection mode, total/subtotal & $22 / 8$ & $9 / 6$ & 0.209 \\
\hline $\begin{array}{l}\text { Intraoperative chemotherapy, no/ } \\
\text { yes }\end{array}$ & $5 / 25$ & $4 / 10$ & 0.592 \\
\hline $\begin{array}{l}\text { Intraoperative blood loss, } \\
\leq 2000 \mathrm{~mL} / 2000 \mathrm{~mL}\end{array}$ & $20 / 10$ & $9 / 6$ & 0.617 \\
\hline Operation time, $\leq 4 \mathrm{~h} />4 \mathrm{~h}$ & $17 / 13$ & $9 / 9$ & 0.782 \\
\hline $\begin{array}{l}\text { Radiotherapy for spinal metastasis, } \\
\text { no/yes }\end{array}$ & $18 / 12$ & $6 / 12$ & 0.220 \\
\hline Bisphosphonate treatment, no/yes & $10 / 20$ & $4 / 10$ & 0.372 \\
\hline Systemic chemotherapy, no/yes & $2 / 28$ & $4 / 9$ & 0.122 \\
\hline Immunotherapy, no/yes & $19 / 11$ & $6 / 9$ & 0.032 \\
\hline Total & 30 & 9 & - \\
\hline
\end{tabular}

Notes: $\mathrm{P}$ values of $<0.05$ are shown in bold. *One patient died I month after surgery, so postoperative Frankel Grade and ECOG-PS were evaluated in the remaining 29 patients at their 2-month follow-up.

\section{Univariate and Multivariate Analysis of Prognostic Factors}

The univariate analysis of the prognostic factors affecting OS is shown in Table 1. Patients with postoperative
ECOG-PS of $0-2$ had a longer OS time than those with the score of $3-5 \quad(\mathrm{P}=0.022)$. OS time significantly increased in patients who received immunotherapy $(\mathrm{P}=0032)$. There were no significant differences in other patient-, tumor-, and treatment-related factors.

The above-mentioned two potential prognostic factors were then submitted to the multivariate Cox regression model, and the results showed that both were independent prognostic factors for OS (Table 2). Postoperative ECOGPS of 3-5 was significantly associated with a higher risk of death $(\mathrm{HR}=3.14, \mathrm{P}=0.016)$. The risk of death was significantly decreased in patients who received immunotherapy $(\mathrm{HR}=0.32, \mathrm{P}=0.016)$. Kaplan-Meier curves of $\mathrm{OS}$ for postoperative ECOG-PS and immunotherapy are shown in Figure 1B and C.

\section{Discussion}

Lung cancer is the most common malignant tumor worldwide. SCLC accounts for $15 \%$ of all lung cancers, with more than 180,000 cases per year. ${ }^{3}$ It is reported that more than a quarter of patients with SCLC developed spinal metastasis, but SCC was identified in only $4 \%$ of patients. ${ }^{13}$ Patients with metastatic SCC caused by SCLC who were candidate for surgical intervention were even rarer and poorly understood. To our knowledge, our study represents the largest series of SCC secondary to SCLC. In this study, 30 consecutive patients with SCLC undergoing surgical treatment for metastatic SCC in the past ten years were reviewed. Our study revealed that urgent decompressive surgery played an important role in improving patients' QoL. Postoperative ECOG-PS and immunotherapy were independent prognostic factors.

Bone metastasis was considered to affect survival negatively. In the series of Kang et al, ${ }^{6} 178$ patients with SCLC at extensive-stage were reviewed, 61 of them had bone metastases at the time of diagnosis. $70.5 \%$ of patients received chemotherapy with or without palliative radiotherapy, 9.8\% received palliative radiotherapy only, and $19.7 \%$ did not receive cancer treatment but supportive care. The results showed that OS of patients with bone metastases was shorter than that of patients without bone metastases (4.13 vs 6.17 months, $\mathrm{p}=0.015)$. Early in 1989 , Goldman et al, ${ }^{13}$ reviewed 24 patients with SCC caused by SCLC. Three patients underwent surgical decompression of the spinal cord and radiotherapy. Fourteen patients received radiotherapy, six in conjunction with dexamethasone. Seven patients were given symptomatic treatment only. The median survival from presentation of SCLC was 33 weeks, but only 6 weeks from SCC. In addition, 

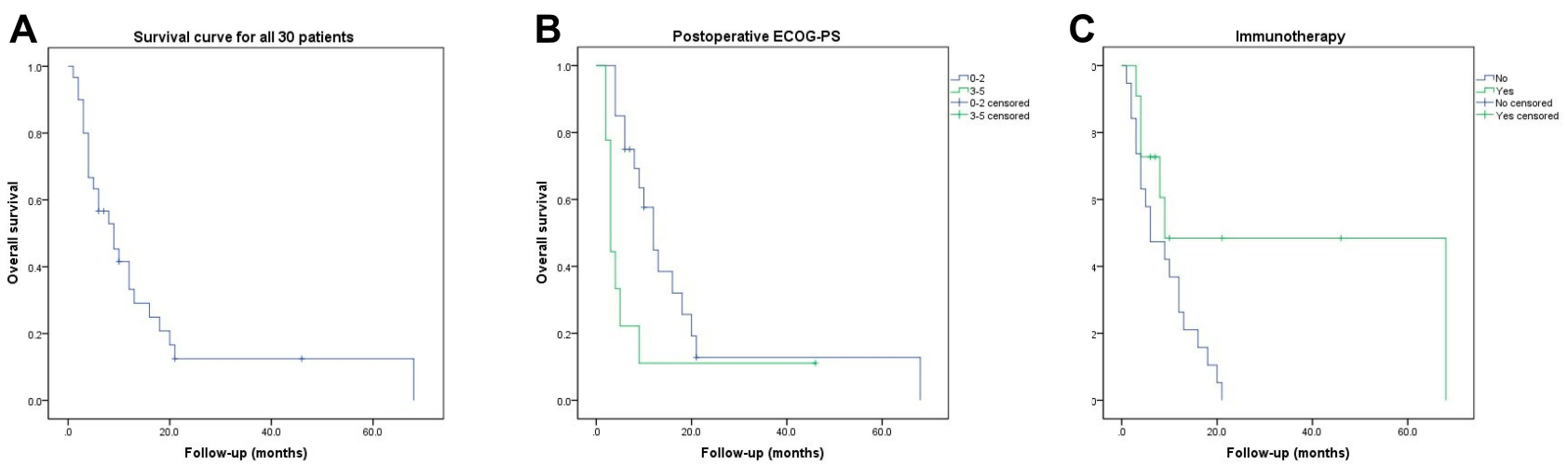

Figure I (A) Kaplan-Meier curve for all patients; (B) and (C) Kaplan-Meier curves of OS for postoperative ECOG-PS (B) and immunotherapy (C).

several cases in the literature of spine or spinal cord metastasis from SCLC were also reviewed, ${ }^{19-27}$ and the main results are shown in Table 3. The survival time of those cases after spinal metastasis ranged from 6 days to 20 months. In our series, the median OS time was 9 months after spinal surgery. Two main reasons may account for the relatively better prognosis in ours. Firstly, the improvement in systematic chemotherapy and development of new therapeutic strategies in past decades contributed to a better overall prognosis in SCLC. ${ }^{28}$ Moreover, a stricter inclusion criterion was followed by us. Only patients who had both a slight systemic burden of tumor and a good performance status to tolerate surgical intervention and systematic therapy were included in our study.

Actually, surgery is not always recommended for spinal metastasis of SCLC. Radiotherapy or radiosurgery could be regarded as an appropriate choice for patients with bone-only disease. However, when it develops to SCC, surgery should be encouraged. In our series, all operative patients were presence of neurological risk, including both current neurological signs or symptoms and potential neurological compromise based on Bilsky epidural SCC scale. Mechanical instability assessed by SINS classification system is another indication for surgery in our series, for pathologic fractures do not respond to radiotherapy and chemotherapy. In addition, although pain control was one of the important goals of surgery, pain itself was not a sufficient prerequisite to performing operation.

Table 2 Multivariate Analysis of Prognostic Factors Affecting OS

\begin{tabular}{|l|l|l|}
\hline Factors & HR (95\% Cl) & P \\
\hline Postoperative ECOG-PS, 0-2/3-5 & $3.14(1.24-7.96)$ & $\mathbf{0 . 0 1 6}$ \\
Immunotherapy, no/yes & $0.32(0.11-0.92)$ & $\mathbf{0 . 0 3 3}$ \\
\hline
\end{tabular}

Note: $P$ values of $<0.05$ are shown in bold.
With the advances in surgical technology and instrumentation, improved outcomes and a broader spectrum of interventions are available to patients with spinal metastatic tumors. Because few patients with extensive-stage disease of SCLC achieve overall treatment response, therapies for symptom control and improved QoL are of particular importance. ${ }^{4}$ In our series, unbearable pain, the most common chief complaint, was relieved substantially in all patients after operation. Improvements in neurological function and performance status were also observed in 10 and 9 patients, respectively. Notably, ambulation ability (Frankel Grade of D-E) was recovered in four patients who preoperatively lost ambulation ability, and five patients regained their self-care ability (ECOG-PS of 0-2) 2 months after surgery. Moreover, patient-reported QoL assessment has been recognized as one of the most important tools for evaluating treatments. In this study, EQ-5D-3L, which have been widely used in clinical trials over 25 years, was selected as a tool to evaluate the QoL from the perspective of patients, and the EQ-5D-3L utility scores showed a significant improvement postoperatively. Therefore, despite the short survival time in SCLC, spinal surgery played a critical role in improving patients' QoL.

For metastatic spine tumor surgery, it is crucial to prevent postoperative complications to ensure that the iatrogenic effects will not exceed its potential benefits. $^{29,30}$ In our series, both patients' and surgeons' aspects were controlled to reduce risk of postoperative complications. On the one hand, strict patient selection was conducted to exclude high-risk patients from surgery. On the other hand, surgeries were performed by specialized surgeons with at least 10-year experience in spine tumor surgery. As a result, only one patient suffered from surgical site infection. Patient-related risk factors for postoperative complications including advanced age, poor nutritional status, comorbidities, prior radiotherapy were 
Table 3 Literature Review of Cases of Spine or Spinal Cord Metastasis from SCLC

\begin{tabular}{|c|c|c|c|c|}
\hline Author & Year & Metastasis Site & Treatment After Spinal Metastasis & $\begin{array}{l}\text { Survival Time After Spinal } \\
\text { Metastasis }\end{array}$ \\
\hline Morita 19 & 2019 & Vertebral & Intensive support & Within a month \\
\hline Osawa ${ }^{20}$ & 2018 & Intramedullary & $\begin{array}{l}\text { Radiotherapy, corticosteroid therapy, and } \\
\text { chemotherapy }\end{array}$ & 20 months \\
\hline Yasui $^{21}$ & 2017 & Epidural & Palliative care & 6 days \\
\hline Xiong $^{22}$ & 2015 & $\begin{array}{l}\text { Extramedullary- } \\
\text { intradural }\end{array}$ & Surgery and chemotherapy & I year \\
\hline Katsenos ${ }^{23}$ & 2013 & Intramedullary & Chemotherapy and radiotherapy & 3 months \\
\hline $\mathrm{Lee}^{24}$ & 2012 & $\begin{array}{l}\text { Extramedullary- } \\
\text { intradural }\end{array}$ & Surgery and chemoradiotherapy & 9 months \\
\hline $\operatorname{Lin}^{25}$ & 2010 & $\begin{array}{l}\text { Extramedullary- } \\
\text { intradural }\end{array}$ & Surgery & I month \\
\hline Koutsis ${ }^{26}$ & 2006 & Intramedullary & Corticosteroid therapy and radiotherapy & I month \\
\hline \multirow[t]{4}{*}{$\begin{array}{l}\text { Murphy }{ }^{27} \\
\text { (4 cases) }\end{array}$} & 1983 & Intramedullary & Radiotherapy & I month \\
\hline & & Intramedullary & Radiotherapy & I month \\
\hline & & Intramedullary & Corticosteroids, radiation, and surgical treatment & 3 months \\
\hline & & Intramedullary & Radiotherapy and chemotherapy & 7 months \\
\hline
\end{tabular}

not observed on this patient. Thus, whether the iatrogenic factors on him, such as preoperative embolization, intraoperative chemotherapy and etc., would influence the likelihood of postoperative complications would be our next research direction.

ECOG-PS is a standard criterion to comprehensively measure and evaluate the living ability of patients. ECOG-PS was reported to be an independent prognostic factor for patients with bone metastasis of SCLC, ${ }^{6}$ and patients with spinal metastasis of NSCLC. ${ }^{11}$ Similarly, our results showed that postoperative ECOG-PS of 0-2 significantly predicted favorable prognosis. Understandably, a good health status was important to enable patients to withstand the following systemic chemotherapy and immunotherapy. Meanwhile, bedridden-related problems (such as increased susceptibility to infection, decubitus ulcer, and deep vein thrombosis) could also be avoided in patients with the ability of ambulation and self-care. Therefore, although the goal of surgery for spinal metastasis is palliative, surgery may improve the survival time indirectly for a proportion of patients, especially who regained their self-care ability, by providing them with better performance statuses.

Immunotherapy, especially immune checkpoint inhibitor, has been revolutionizing cancer treatments and has been an active area of investigation in SCLC as well. PD-1 immune checkpoint inhibitor antibodies nivolumab $^{31}$ and pembrolizumab ${ }^{32,33}$ exhibited promising efficacy for SCLC and were available to Chinese patients.
In our series, 11 patients received immunotherapy of PD-1 immune checkpoint inhibitor after completion of chemotherapy in department of medical oncology. Our results showed that OS time increased in patients who received immunotherapy (9 months vs 6 months), and multivariate analysis confirmed that immunotherapy was an independent prognostic factor.

SCLC is very chemosensitive and, therefore, the standard treatment for patients with SCLC is combination chemotherapy. $^{3,34}$ Unfortunately, although $35-86 \%$ of patients respond to first-line chemotherapy of platinumetoposide doublet, disease relapses rapidly, and outcomes with second-line treatment of topotecan or amrubicin are poor. $^{31}$ In our series, intraoperative chemotherapy was used for selected patients under the hypothesis that local accumulation of anticancer agents leads to enhanced efficacy with decreased systemic toxicity. Postoperatively, all patients were transferred to department of medical oncology, and received chemotherapy performed by our multidisciplinary team except for the two who had the poor general condition after surgery. Radiotherapy represents the major treatment of patients with spinal metastases. New advances in radiation technology, such as spine stereotactic body radiotherapy, have allowed delivery of higher doses of radiation to the target volume with minimal injury to surrounding tissue. In our series, because the presence of SCC was oncological emergency, prompt surgical decompression rather than radiotherapy was the first 
choice of treatment. Radiotherapy was performed postoperatively in 12 patients. Our results showed that a longer OS was achieved in both patients who received systemic chemotherapy (9 months vs 4 months) and patients who received postoperative radiotherapy (12 months vs 6 months). But largely due to the limited sample size, these differences were not statistically significant. Other therapies, such as bisphosphonate treatment, surgery and radiotherapy for lung were also failed to show their significant improvements for OS in our series, and their efficacy needs further investigations.

Several limitations of this study should be mentioned. Firstly, the retrospective nature is the main limitation. Secondly, all patients enrolled in this series received surgical intervention. There was no control group treated without surgery. Thirdly, we did not design subgroups for analyses of chemotherapy and immunotherapy based on detailed therapeutic schemes due to the limited sample size. Fourthly, it is also because of the small sample size, we failed to analyze the risk factors for postoperative complications in depth.

\section{Conclusions}

In conclusion, through analysis of 30 consecutive patients surgically treated for metastatic SCC from SCLC, we concluded that despite the short life expectancy, prompt surgical decompression is extremely necessary for patients with SCC caused by SCLC, for surgery played a critical role in improving patients' QoL. Postoperative ECOG-PS of 0-2 and immunotherapy were identified to be favorable prognostic factors for OS. Consideration of these findings might be helpful in treating patients with metastatic SCC from SCLC. A promising outcome might be achieved by timely surgical intervention followed by standard chemotherapy and further immunotherapy for selected patients.

\section{Funding}

This work was supported by key project funding in the basic research field of the Shanghai Municipal Science and Technology Commission (17JC1400903).

\section{Disclosure}

The authors declare that they have no conflicts of interest in this work.

\section{References}

1. Gazdar AF, Bunn PA, Minna JD. Small-cell lung cancer: what we know, what we need to know and the path forward. Nat Rev Cancer. 2017;17(12):725-737. doi:10.1038/nrc.2017.87
2. Pietanza MC, Byers LA, Minna JD, Rudin CM. Small cell lung cancer: will recent progress lead to improved outcomes? Clin Cancer Res. 2015;21(10):2244-2255. doi:10.1158/1078-0432.CCR14-2958

3. van Meerbeeck JP, Fennell DA, De Ruysscher DK. Small-cell lung cancer. Lancet. 2011;378(9804):1741-1755. doi:10.1016/S01406736(11)60165-7

4. Zikos E, Ghislain I, Coens C, et al. Health-related quality of life in small-cell lung cancer: a systematic review on reporting of methods and clinical issues in randomised controlled trials. Lancet Oncol. 2014;15(2):e78-89. doi:10.1016/S1470-2045(13)70493-5

5. Jackman DM, Johnson BE. Small-cell lung cancer. Lancet. 2005;366 (9494):1385-1396. doi:10.1016/S0140-6736(05)67569-1

6. Kang EJ, Lee SY, Kim HJ, et al. Prognostic Factors and Skeletal-Related Events in Patients with Small Cell Lung Cancer with Bone Metastases at the Time of Diagnosis. Oncology. 2016;90 (2):103-111. doi:10.1159/000442949

7. Prasad D, Schiff D. Malignant spinal-cord compression. Lancet Oncol. 2005;6(1):15-24. doi:10.1016/S1470-2045(05)70022-X

8. Bartels RH, van der Linden YM, van der Graaf WT. Spinal extradural metastasis: review of current treatment options. CA Cancer J Clin. 2008;58(4):245-259. doi:10.3322/CA.2007.0016

9. Patchell RA, Tibbs PA, Regine WF, et al. Direct decompressive surgical resection in the treatment of spinal cord compression caused by metastatic cancer: a randomised trial. Lancet. 2005;366 (9486):643-648. doi:10.1016/S0140-6736(05)66954-1

10. Fehlings MG, Nater A, Tetreault L, et al. Survival and Clinical Outcomes in Surgically Treated Patients With Metastatic Epidural Spinal Cord Compression: Results of the Prospective Multicenter AOSpine Study. J Clin Oncol. 2016;34(3):268-276. doi:10.1200/ JCO.2015.61.9338

11. Tang Y, Qu J, Wu J, Li S, Zhou Y, Xiao J. Metastatic Spinal Cord Compression from Non-Small-Cell Lung Cancer Treated with Surgery and Adjuvant Therapies: A Retrospective Analysis of Outcomes and Prognostic Factors in 116 Patients. J Bone Joint Surg Am. 2015;97(17):1418-1425. doi:10.2106/JBJS.N.01124

12. Tang Y, Qu J, Wu J, et al. Effect of Surgery on Quality of Life of Patients with Spinal Metastasis from Non-Small-Cell Lung Cancer. J Bone Joint Surg Am. 2016;98(5):396-402. doi:10.2106/JBJS. O.00629

13. Goldman JM, Ash CM, Souhami RL, et al. Spinal cord compression in small cell lung cancer: a retrospective study of 610 patients. $\mathrm{Br}$ J Cancer. 1989;59(4):591-593. doi:10.1038/bjc.1989.119

14. Laufer I, Rubin DG, Lis E, et al. The NOMS framework: approach to the treatment of spinal metastatic tumors. Oncologist. 2013;18 (6):744-751. doi:10.1634/theoncologist.2012-0293

15. Barzilai O, Laufer I, Yamada Y, et al. Integrating Evidence-Based Medicine for Treatment of Spinal Metastases Into a Decision Framework: Neurologic, Oncologic, Mechanicals Stability, and Systemic Disease. J Clin Oncol. 2017;35(21):2419-2427. doi:10.1200/JCO.2017.72.7362

16. Fisher CG, DiPaola CP, Ryken TC, et al. A novel classification system for spinal instability in neoplastic disease: an evidence-based approach and expert consensus from the Spine Oncology Study Group. Spine. 2010;35(22):E1221-1229. doi:10.1097/BRS.0b013e3181e16ae2

17. Bilsky MH, Laufer I, Fourney DR, et al. Reliability analysis of the epidural spinal cord compression scale. J Neurosurg Spine. 2010;13 (3):324-328. doi:10.3171/2010.3.SPINE09459

18. Liu GG, Wu H, Li M, Gao C, Luo N. Chinese time trade-off values for EQ-5D health states. Value Health. 2014;17(5):597-604. doi:10.1016/j.jval.2014.05.007

19. Morita S, Suda T, Oda C, et al. The Value of (18)F-FDG PET in the Diagnosis of Intertrabecular Vertebral Metastasis in a Small Cell Lung Cancer Patient with a High Serum CEA Level. Intern Med. 2019;58(3):415-418. doi:10.2169/internalmedicine.1394-18 
20. Osawa H, Okauchi S, Ohara G, Kagohashi K, Satoh H. A Long-Term Control of Intramedullary Thoracic Spinal Cord Metastasis from Small Cell Lung Cancer. Acta Medica. 2018;61(2):57-59. doi:10.14712/18059694.2018.52

21. Yasui H, Ozawa N, Mikami S, et al. Spinal Cord Ischemia Secondary to Epidural Metastasis from Small Cell Lung Carcinoma. Am J Case Rep. 2017;18:276-280. doi:10.12659/AJCR.902813

22. Xiong J, Zhang P. Cauda equina syndrome caused by isolated spinal extramedullary-intradural cauda equina metastasis is the primary symptom of small cell lung cancer: a case report and review of the literature. Int J Clin Exp Med. 2015;8(6):10044-10050.

23. Katsenos S, Nikolopoulou M. Intramedullary thoracic spinal metastasis from small-cell lung cancer. Monaldi Arch Chest Dis. 2013;79 (3-4):140-142. doi:10.4081/monaldi.2013.5214

24. Lee CH, Kim KJ, Hyun SJ, Jahng TA, Kim HJ. Intradural extramedullary metastasis of small cell lung cancer: a case report. Korean J Spine. 2012;9(3):293-296. doi:10.14245/kjs.2012.9.3.293

25. Lin CL, Chang JL, Lo HC, Wu KA. Extramedullary-intradural spinal metastasis of small cell lung cancer causing cauda equina syndrome. Am J Med Sci. 2010;339(2):192-194. doi:10.1097/ MAJ.0b013e3181bedd1f

26. Koutsis G, Spengos K, Potagas C, Dimitrakopoulos A, Sfagos K, Zakopoulos N. Intramedullary spinal cord metastases in a patient with small-cell lung cancer. Eur J Intern Med. 2006;17(5):372-374. doi:10.1016/j.ejim.2005.12.014

27. Murphy KC, Feld R, Evans WK, et al. Intramedullary spinal cord metastases from small cell carcinoma of the lung. J Clin Oncol. 1983;1(2):99-106. doi:10.1200/JCO.1983.1.2.99
28. Zimmerman S, Das A, Wang S, Julian R, Gandhi L, Wolf J. 2017-2018 Scientific Advances in Thoracic Oncology: Small Cell Lung Cancer. J Thorac Oncol. 2019;14(5):768-783. doi:10.1016/j. jtho.2019.01.022

29. Zairi F, Karnoub MA, Vieillard MH, et al. Evaluation of the relevance of surgery in a retrospective case series of patients who underwent the surgical treatment of a symptomatic spine metastasis from lung cancer. Eur Spine J. 2016;25(12):4052-4059. doi:10.1007/ s00586-016-4397-4

30. Bouras T, Zairi F, Arikat A, Vieillard MH, Allaoui M, Assaker R. Decision Making for the Surgical Treatment of Vertebral Metastases Among Patients with Short Predicted Survival. World Neurosurg. 2018;111:e573-e580. doi:10.1016/j.wneu.2017.12.107

31. Antonia SJ, Lopez-Martin JA, Bendell J, et al. Nivolumab alone and nivolumab plus ipilimumab in recurrent small-cell lung cancer (CheckMate 032): a multicentre, open-label, Phase 1/2 trial. Lancet Oncol. 2016;17(7):883-895. doi:10.1016/S1470-2045(16)30098-5

32. Ott PA, Elez E, Hiret S, et al. Pembrolizumab in Patients With Extensive-Stage Small-Cell Lung Cancer: Results From the Phase Ib KEYNOTE-028 Study. J Clin Oncol. 2017;35(34):3823-3829. doi:10.1200/JCO.2017.72.5069

33. Gadgeel SM, Pennell NA, Fidler MJ, et al. Phase II Study of Maintenance Pembrolizumab in Patients with Extensive-Stage Small Cell Lung Cancer (SCLC). J Thorac Oncol. 2018;13 (9):1393-1399. doi:10.1016/j.jtho.2018.05.002

34. Waqar SN, Morgensztern D. Treatment advances in small cell lung cancer (SCLC). Pharmacol Ther. 2017;180:16-23. doi:10.1016/j. pharmthera.2017.06.002

\section{Publish your work in this journal}

Cancer Management and Research is an international, peer-reviewed open access journal focusing on cancer research and the optimal use of preventative and integrated treatment interventions to achieve improved outcomes, enhanced survival and quality of life for the cancer patient.
The manuscript management system is completely online and includes a very quick and fair peer-review system, which is all easy to use. Visit http://www.dovepress.com/testimonials.php to read real quotes from published authors. 